7-9-2013

\title{
Classification and Volumetric Analysis of Temporal Bone Pneumatization Using Cone Beam Computed Tomography
}

Aniket B. Jadhav

UCHC School of Dental Medicine, dr.aniket.jadhav@gmail.com

\section{Recommended Citation}

Jadhav, Aniket B., "Classification and Volumetric Analysis of Temporal Bone Pneumatization Using Cone Beam Computed Tomography" (2013). Master's Theses. 460.

https://opencommons.uconn.edu/gs_theses/460 


\title{
Classification and Volumetric Analysis of Temporal Bone Pneumatization Using Cone Beam Computed Tomography
}

\begin{abstract}
Aniket Jadhav
B.D.S., MGV's KBH Dental College and Hospital- India 2008.
\end{abstract}

\author{
A Thesis \\ Submitted in Partial Fulfillment of the \\ Requirements for the Degree of \\ Master of Dental Science \\ At the \\ University of Connecticut
}

2013 


\begin{abstract}
APPROVAL PAGE
Masters of Dental Science Thesis

Classification and Volumetric Analysis of Temporal Bone Pneumatization Using Cone Beam Computed Tomography
\end{abstract}

\begin{tabular}{l} 
Presented by \\
Aniket Jadhav, B.D.S. \\
Major Advisor_ \\
Associate Advisor_ $\quad$ Dr. Alan G. Lurie \\
\hline Associate Advisor_ $\quad$ Dr. Douglas Fellows \\
\hline Associate Advisor_ \\
\hline Dr. Arthur R. Hand \\
\hline
\end{tabular}

Dr. Aditya Tadinada

University of Connecticut

2013 


\section{Acknowledgment}

I take this opportunity to express my gratitude to the people who have been instrumental in the successful completion of this project.

I would like to show my greatest appreciation to Dr. Alan Lurie. I can't say thank you enough for his tremendous support and help. Without his encouragement and guidance this project would not have materialized. The guidance and support received from all the members who contributed and who are contributing to this project, was vital for the success of the project. I am grateful for their constant support and help I would like to thank my committee members, Dr. Douglas Fellows, Dr. Arthur Hand and Dr. Aditya Tadinada for their encouragement and insightful comments. Furthermore I would also like to acknowledge with much appreciation the crucial role of Dr. Kandasamy Rengasamy who gave me permission to use all required data for this project. Finally I would like to thank my parents for supporting my education at this premier institute and for their continued encouragement. 


\section{TABLE OF CONTENTS}

Title Page $\quad$ i

Approval Page $\quad$ ii

Acknowledgment iii 
Table of Contents $\quad$ iv

1. Introduction 1

2. Objective and Hypothesis 3

3. Materials and Methods 4

4. Data and Statistical Analysis 10

$\begin{array}{lll}\text { 5. Results } & 11\end{array}$

$\begin{array}{lll}\text { 6. Discussion } & 14\end{array}$

$\begin{array}{lll}\text { 7. Conclusion } & 18\end{array}$

8. Future Directions 19

$\begin{array}{lll}9 . & \text { References } & 20\end{array}$ 


\section{Introduction:}

The advent of Cone Beam Computed Tomography (CBCT) over the past decade, with its applications in dentistry in general and Oral and Maxillofacial Radiology (OMFR) in particular, has led to a variety of maxillofacial applications, including evaluation of portions of the skull base. During routine OMF radiology practice, portions of the skull base that include the temporal bone are visualized. The temporal bone often presents a varied pneumatization pattern that has a specific clinical significance especially important for planning any surgical procedures in this area.

Each temporal bone consists of four components: the squamous, petromastoid and tympanic parts and the styloid processes [10]. A proposed function of aeration includes resonance, acting as a reservoir of air for the middle ear to compensate for altered function of the Eustachian tube (ET), thereby preventing negative pressure, and avoiding changes in middle ear mucosa which may progress to otitis media $[18,29]$.

Pneumatization is the process whereby epithelium infiltrates the developing bone and forms epithelial lined air cell cavities [14]. The squamous, petromastoid and tympanic parts are the most frequently pneumatized parts $[5,1,32]$, but pneumatization may be extend to the articular eminence $[32,7]$ of the zygomatic process. The environmental theory of Wittmaack suggests that middle ear mucosa is a pre-requisite for normal pneumatization while Diamant suggests that pneumatization is genetically dependent. The pneumatization process usually begins prenatally, during the 22nd-24th gestational weeks. At around 28 weeks of gestation, the petrous apex begins to pneumatize [20]. The pattern of pneumatization of the temporal bone is usually completed by the age of 10 years in females and 15 years in males [9].

Numerous studies have reported on the variety of pneumatization patterns of the temporal bone and their classifications using computed tomography (CT). Although CT has several advantages over CBCT, especially for depicting soft tissues, comparable evaluation of the osseous 
components of the skull base and sinonasal anatomy are possible at a lower radiation dose and at a lower cost with CBCT.

In routine OMF radiology practice, a bulk of the temporal bone is routinely visualized on TMJ and orthodontic studies; thus, it is important for dentists in general and oral radiologists in particular to know normal anatomical variations and pneumatization patterns to identify any pathological changes and make appropriate referrals to specialists in this region like otorhinolaryngologists and neuroradiologists. 


\section{Objective and Hypothesis}

Objective: To classify and characterize the pneumatization patterns of the temporal bone using CBCT data sets.

Specific Aim: To perform volumetric analysis and classify different repeated patterns of temporal bone pneumatization in adults using CBCT data sets.

Hypothesis: Cone Beam Computed Tomography can be used for qualitative and quantitative assessment of pneumatization patterns of the temporal bone in adults. 


\section{Materials and Methods:}

\section{Data Collection:}

Data for the study were gathered after an approval from the Institutional Review Board (IRB) of The UCONN Health Center was obtained for the study. (IRB \# 13-120-1.)

One hundred and fifty five temporal bones were analyzed from 78 patients who were asymptomatic for any oro-nasal pathology.

The inclusion and exclusion criteria for this retrospective analysis of CBCT scans were as follows (Table 1).

1. Patients above the age of 18 and not over the age of 70 years.

2. All the scans with a field of view (FOV) more than eight inches in diameter.

The inclusion criteria for the selected scans consist of complete visualization of external auditory canal, middle ear, Perilabyrinthine area and articular eminence. Cases with presence of soft tissue, fluid levels, bone sclerosis and destruction and any radiographic evidence of surgery were excluded (Table 1).

Table 1: Inclusion and Exclusion Criteria

\begin{tabular}{|l|ll|}
\hline Inclusion Criteria & Exclusion Criteria \\
\hline - Complete visualization of: & $\bullet$ Soft tissue and/or fluid \\
- Middle ear & levels \\
- Perilabyrinthine region & - Sclerosis \\
- Petrous apex & Destruction of the bony \\
- Articular eminence & margins \\
& - Radiographic evidence of \\
& surgical procedure \\
\hline
\end{tabular}




\section{Analysis of the Scans:}

There are three primary regions where pneumatization in the temporal bone can be seen: mastoid, perilabyrinthine and the petrous apex. There are numerous studies which looked at mastoid pneumatization due to their large air cells based on Multi-slice CT (MSCT) and conventional radiography. In this study we limited the assessment of the pneumatization pattern to the middle ear, perilabyrinthine and petrous apex regions because the majority of large field of view scans of this region show only a part of the mastoid air cells. The mastoid air cells are not included en toto in the large volume CBCT scanning protocols because it is usually not the specific area of interest. Both sides of the skull base were assessed for quantitative and qualitative analysis using CB works 3.0 (Hitachi Medical System America, Inc.) DICOM viewer. To see the greatest area of pneumatization, a correction of the Z-axis was done in such way that the malleoincudal complex appeared as an ice-cream cone shaped structure on axial images.

\section{Establishing reference structures for simple classification of temporal bone pneumatization:}

This study followed the method of selection of the reference structures from a study conducted by Han et al. [12]. Temporal bone pneumatization is classified into the three groups using labyrinthine and petrous segment of the internal carotid canal as reference structures.

Labyrinthine is used as a reference structure to classify the pneumatization of the temporal bone around the inner ear structures using the following grouping:

- Group 1: No evidence of pneumatization in the region of inner ear (Fig 1A).

- Group 2: Pneumatization present either medial or lateral to the superior semicircular canal on axial section (Fig 1B).

- Group 3: Peri- labyrinthine pneumatization (Fig 1C). 


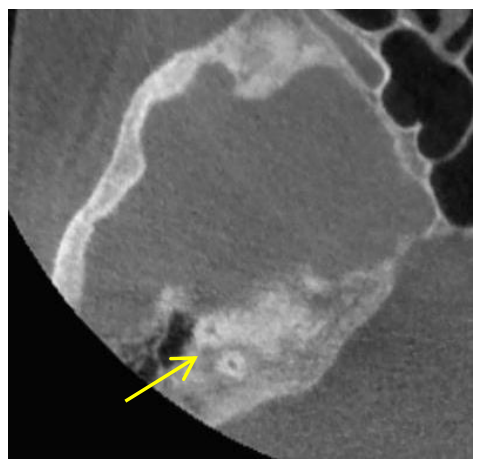

Fig 1

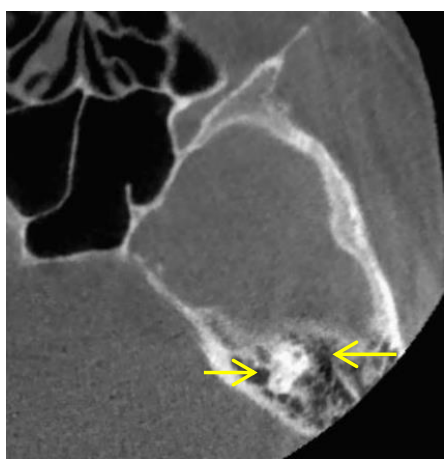

$\mathrm{B}$

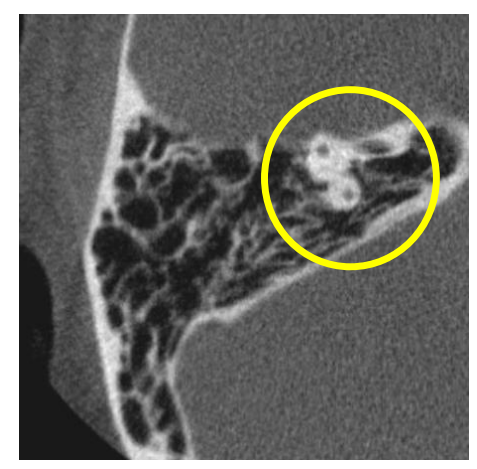

$\mathrm{C}$

Petrous segment of Internal Carotid Canal is used for assessing the pneumatization of the petrous apex.

- Group 1: No pneumatization of petrous apex (Fig 2A).

- Group 2: (Mild pneumatization of petrous apex); there are irregularly evident small numbers of air cells on either side (medial or lateral) of the carotid canal (Fig 2B).

- Group 3: (Complete Pneumatization) of petrous apex; pneumatization is present surrounding the carotid canal (Fig 2C).

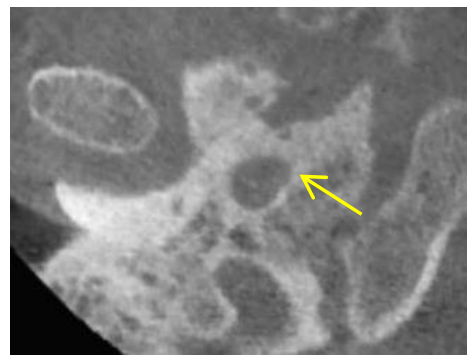

Fig 2

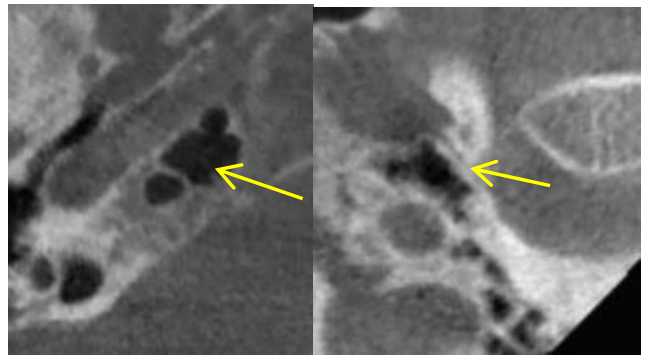

B

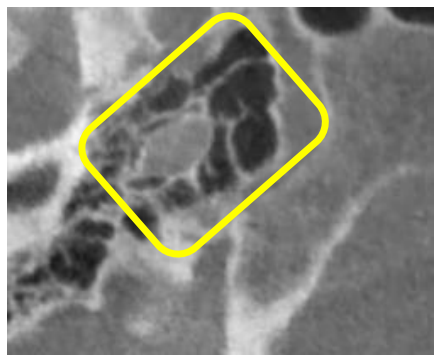

$\mathrm{C}$ 
Direct communication of peritubal cells with ET was also assessed to assess possible correlation with degree of pneumatization:

Presence of peritubal cells was assessed to see its correlation with degree of pneumatization of the temporal bone and further incidence of direct communication (Fig. 3) of peritubal cells with the bony portion of the eustachian tube was also analyzed.

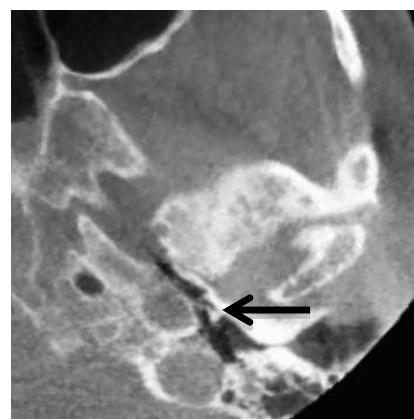

A

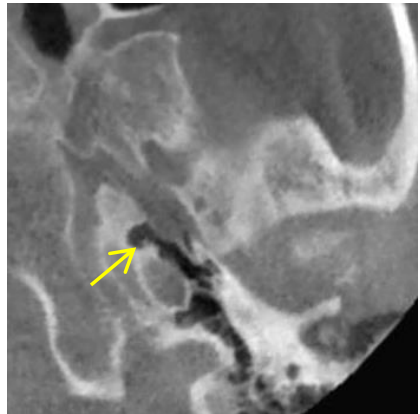

B

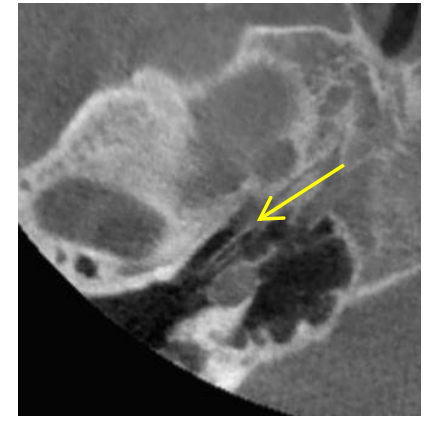

$\mathrm{C}$

Fig 3: Showing direct communication of peritubal cell with bony portion of ET.

\section{Calculation of Volume:}

Pneumatization in the temporal bone is mainly present in the mastoids, perilabyrinthine region, middle ear/tympanic cavity and petrous part of the temporal bone. Since CBCT utilizes isotropic voxels with thin slices $(0.2 \mathrm{~mm}-0.4 \mathrm{~mm})$, it can precisely locate and measure small pneumatized spaces like the Perilabyrinthine region, peritubal region and petrous apex. This is the first type of study in the English literature looking at pneumatization in the middle ear, perilabyrinthine and petrous apex region only using a CBCT data set. Measurements of pneumatization of the temporal bone included the spaces of:

- Middle ear cavity including eustachian tube;

- Perilabyrinthine region;

- Petrous apex. 
The acquired data were stored in DICOM format (Digital imaging and communication in medicine) and imported into the CB Works 3.0 (Hitachi Medical System America Inc).

The slice thicknesses and slice distances varied from $0.2 \mathrm{~mm}$ to $0.4 \mathrm{~mm}$. Images were segmented using lower and upper window levels of -1024 and -290 pixel intensity values to mark the pneumatized spaces. The windowing function in computed tomography enables the operator to narrow the shades of gray. The human eye is capable of distinguishing 32 shades of gray while CT data has a range of 4,096 shades of gray per pixel. In this study we selected the window threshold of -1024 and -290 pixel intensity to accurately mark the air cavities. In the thresholding process, the window width and level settings map the measured attenuation of each voxel to a corresponding gray scale value to appropriately mark the tissue of interest.

All other regions such as pneumatization in the articular eminence, part of sphenoid sinus and the external auditory canal were excluded using the region of interest tool (Fig. 4). In Fig. 4A, the initial process of thresholding shows marking of all air cavities in the craniofacial area which includes paranasal sinuses, nasal cavity, air cells in the skull base/pneumatized bone in the skull base and airway. As with MSCT data, CBCT data sets can be easily used to perform thresholding and segmentation of bone and air cavities.

Once all the air cavities are marked, the region of interest tool is applied. Since CBCT utilizes isotropic voxels, multiplanar views (axial, sagittal and coronal) which precisely mark the area of interest can be used to limit or extend the area of volume measurement accordingly (Fig. 4B). Once air spaces are selected, the software algorithm calculates the volume of area of interest (Fig. 4C). 
Window thresholding to mark the pneumatized spaces

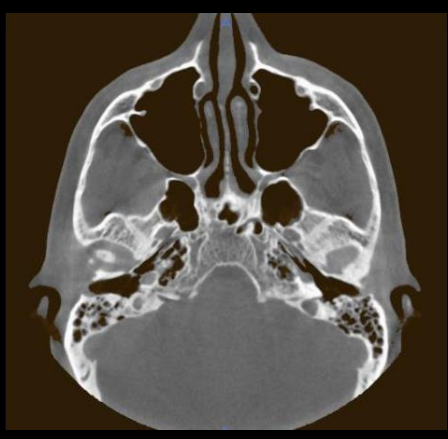

Marking region of Interest

Other regions such as articular eminence, sphenoid sinus and external auditory canal were excluded.

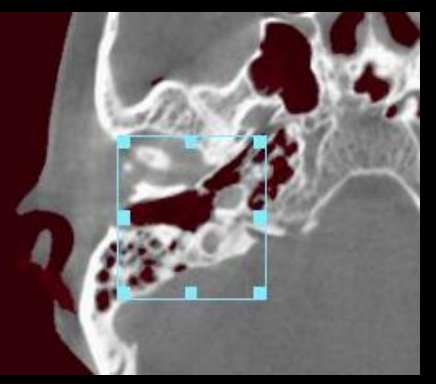

Selected air spaces

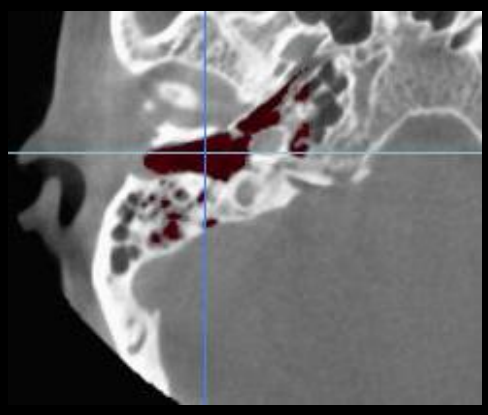




\section{Data and Statistical Analysis:}

Data analysis to evaluate the pattern of pneumatization was done by one OMF radiology faculty member (AT) and one third year OMF radiology resident (AJ). Differences in the volumes of temporal bone pneumatization according to the degree of pneumatization between the groups for each reference structure were investigated using one way analysis of variance (ANOVA) and a post hoc test. Student's $t$-Test was performed to assess possible differences in volume between right and left temporal bones in the same subject. Correlation of direct communication of peritubal cells to ET was analyzed using Pearson's correlation. All statistical analyses were done using SPSS version 21 [17]; $p$ values less than 0.05 were considered statistically significant. 


\section{Results:}

This study population consisted of 78 patients; 27 males and 51 females with age ranges from 18 to 70 years. A total of 155 temporal bones were analyzed to classify the pattern of pneumatization; 78 on the right side and 77 on the left side. The mean volume of temporal bone pneumatization was $1337+/-9 \mathrm{~mm}^{3}$. The difference between right and left sides was not statistically significant $(p=0.930)$.

\section{Classification of temporal bone pneumatization and its corresponding volumetric analysis}

\section{Evaluation of temporal bone using labyrinthine as a reference structure:}

Cases were classified into three groups. Group 1 consisted of 62 cases, with a mean volume of $822 \mathrm{~mm}^{3}$ and a volume range of $450 \mathrm{~mm}^{3}$ to $1325 \mathrm{~mm}^{3}$. Since group 1 indicates no pneumatization of the temporal bone, the calculated value represents the volume of the middle ear cavity. Group 2 consisted of 41 cases with a mean volume of $1269 \mathrm{~mm}^{3}$ and a volume range of $760 \mathrm{~mm}^{3}$ to $2185 \mathrm{~mm}^{3}$. Group 3 consisted of 52 cases with a mean volume of $2008 \mathrm{~mm}^{3}$ and a volume range of $1010 \mathrm{~mm}^{3}$ to $3050 \mathrm{~mm}^{3}$.

Overlapping of volumetric measurements between three groups in few cases were observed in this study due to the large size of the air cells. Despite this, cases were segregated according to their extension and not based on their volumetric measurements.

Using ANOVA, the three groups showed statistically significant differences $(\mathrm{p}<0.0001)$ in their mean volumes (Fig. 5A). A post hoc test was performed to see the source of significant differences in the groups. Significant differences existed between all groups $(p<0.0001)$ [Table $2]$.

\section{Evaluation of temporal bone using carotid canal as a reference structure}

Cases were again classified into three groups. Group 1 consisted of 51 cases with a mean volume of $781 \mathrm{~mm}^{3}$ and a volume range of $443 \mathrm{~mm}^{3}$ to $1325 \mathrm{~mm}^{3}$. Since group 1 indicates no pneumatization of the temporal bone, the calculated value represents the volume of the middle ear cavity. Group 2 consisted of 57 cases with a mean volume of $1268 \mathrm{~mm}^{3}$ and a volume range 
of $840 \mathrm{~mm}^{3}$ to $2780 \mathrm{~mm}^{3}$. Group 3 consisted of 47 cases with a mean volume of $2033 \mathrm{~mm}^{3}$ and a volume range of $1178 \mathrm{~mm}^{3}$ to $3021 \mathrm{~mm}^{3}$.

Overlapping of volumetric measurements between three groups were observed in this study due to the large size of the air cells in few cases. Despite this, cases were segregated according to their extension and not based on their volumetric measurements.

Using ANOVA, the three groups showed statistically significant differences $(\mathrm{p}<0.0001)$ in their mean volumes (Fig 5B). The post-hoc test showed statistically significant differences between the volumes of all three groups $(\mathrm{p}<0.0001)$ (Table 2).

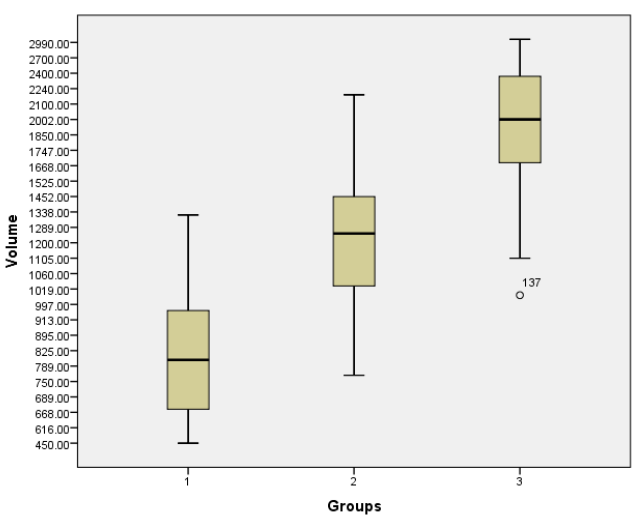

A) Labyrinth as a Reference Structure

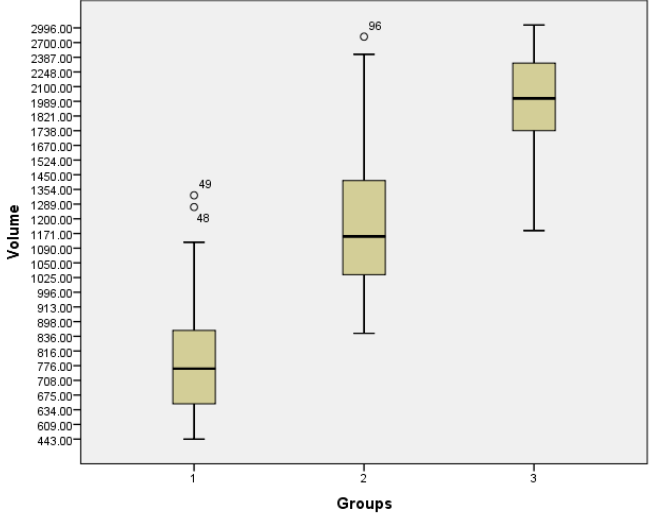

B) Carotid Canal as a Reference Structure

Fig: 5 Pneumatization volumetric measurements of the three groups using Labyrinthine and carotid canal as reference structures showed significant differences in their mean volumes.

\section{Difference in volume of temporal bone between reference structures:}

No statistically significant differences $(\mathrm{p}=0.966)$ exist between the volumes of the temporal bone when calculated using two reference structures (Table 2). This indicates that either of the reference structures can be successfully used to classify and calculate the volume of temporal bone pneumatization. 
Table 2: Mean volume of temporal bone.

\begin{tabular}{|c|c|c|c|c|}
\hline & Labyrinthine as reference & $\begin{array}{c}\text { Carotid canal as a } \\
\text { reference }\end{array}$ & ANOVA & Post Hoc \\
\hline Group 1 & $\begin{array}{c}822 \mathrm{~mm}^{3} \\
\text { SD: } 184.61 \mathrm{~mm}^{3}\end{array}$ & $\begin{array}{c}781 \mathrm{~mm}^{3} \\
\mathrm{SD}: 177.2 \mathrm{~mm}^{3}\end{array}$ & \multirow{3}{*}{$\mathrm{P}<0.0001$} & \multirow{3}{*}{$\mathrm{P}<0.0001$} \\
\hline Group 2 & $\begin{array}{c}1269 \mathrm{~mm}^{3} \\
\text { SD: } 294.5 \mathrm{~mm}^{3}\end{array}$ & $\begin{array}{c}1268 \mathrm{~mm}^{3} \\
\text { SD: } 391.9 \mathrm{~mm}^{3}\end{array}$ & & \\
\hline Group 3 & $\begin{array}{c}2008 \mathrm{~mm}^{3} \\
\text { SD: } 516.3 \mathrm{~mm}^{3}\end{array}$ & $\begin{array}{c}2033 \mathrm{~mm}^{3} \\
\text { SD: } 477.3 \mathrm{~mm}^{3}\end{array}$ & & \\
\hline t -Test & \multicolumn{2}{|c|}{$\mathrm{P}=0.966$} & & \\
\hline
\end{tabular}

Presence of peritubal cells and its correlation with degree of pneumatization:

Of the 155 temporal bones, 99 temporal bones (63.9\%) showed the presence of peritubal cells while $56(36.1 \%)$ had no evidence of peritubal cells. A statistically significant correlation ( $\mathrm{p}<$ 0.0001) was found between degree of pneumatization and presence of peritubal cells. The presence of peritubal cells was further categorized on the basis of direct communication with the bony part of the eustachian tube. Direct communication of peritubal cells at the lateral aspect of the bony segment of the eustachian tube occurred in $41.3 \%$ of the temporal bones. Communication on the medial aspect was seen in $12.3 \%$ of the temporal bones, while $10.3 \%$ showed communication at the anterior third of the bony segment of the eustachian tube. 


\section{Discussion:}

Cone Beam CT technology, also known as volumetric tomography, was developed in the early 1970s and was then utilized in vascular imaging until the early 1980s. This initial prototype was based on a C-arm instrument. In 1995, two Italian inventors introduced CBCT to dentistry and the New Tom DVT 9000 was launched in European markets in 1999 [28]. Apart from dentistry, this technology has gained popularity in the field of otolaryngology, especially for imaging of paranasal sinuses and the temporal bone.

CBCT is based on a rotating gantry with a fixed source of $\mathrm{x}$-rays and a detector. During rotation multiple planar projection images are acquired in an arc of more than 180 degrees. CBCT technology utilizes a cone shaped beam vs. a fan shaped beam in computed tomography. Orthogonal planar images are secondarily reconstructed from multiple basis projection while in computed tomography, primary reconstruction produces axial images and then secondary reconstruction generates orthogonal slices.

To date, numerous studies have been published on uses of CBCT technology for imaging of the temporal bone, specifically middle and inner ear structures [16, 21, 22, 25, and 34]. CBCT is not only being used in pre-surgical evaluation of the temporal bone, it is also now being tested intraoperatively for surgery of the skull base and the temporal bone. Multiple commercial CBCT systems are available, from limited fields of view to large fields of view. Most of the new generation CBCT machines give the operator flexibility to choose from various FOV's targeted to the area of interest.

Pneumatization in the temporal bone is divided into five major regions; middle ear, mastoid, petrous apex, perilabyrinthine and accessory. In this study we examined pneumatization in the middle ear, perilabyrinthine and the petrous apex regions only. In maxillofacial CBCT imaging, these are the primary regions that usually appear on large field of view scans, as opposed to dedicated temporal bone studies which include the entire temporal bone. Pneumatic spaces in the tympanic cavity arise independently of the mastoid antrum, while perilabyrinthine and petrosal apex cells originate from extensions of the mastoid air cells. The supralabyrinthine region is pneumatized by the posterior superior and subarcuate tracts while the infralabyrinthine region is 
pneumatized by hypotympanum, posteromedial and peritubal cells. The petrous apex is pneumatized by cells from the perilabyrinthine region and peritubal tracts [33].

There are numerous studies of pneumatization of the temporal bone and its volumetric analysis using conventional radiography and computed tomography. A study conducted by Han et al. [12] showed a mean volume of $15.28+/-5.34 \mathrm{~cm}^{3}$ using computed tomography. Stieglitz [29] reported a mean petrous bone air cell volume of $10.97 \mathrm{ml}$ based on MSCT imaging. In our study the mean volume of temporal bone, was $1337 \mathrm{~mm}^{3}$ which is relatively less than these two studies because we did not include the mastoid air cell system in the classification system and its volumetric analysis.

In this study we used two reference structures and classified the pneumatization into three groups. By grouping pneumatization, we were able to show the repeated patterns of extension of air cells in the temporal bone. In regard to reference structures, labyrinthine and carotid canal are easy to locate anatomical landmarks on CBCT scans. The numbers of cases in each group are different for both the reference structures but the calculated mean volume for each group is not statistically different (Table 2). In this study we showed that either of the reference structures can be used to classify and perform volume analysis of the pneumatization pattern in the temporal bone.

CBCT utilizes isotropic voxels with slice thickness of less than $0.4 \mathrm{~mm}$ and has higher spatial resolution than MSCT. This makes CBCT technology superior to MSCT when looking at bone and the air cavities where high contrast resolution is not indicated. In the present study we calculated the volume of temporal bone air cells including the middle ear cavity on CBCT data. Group 1 which represents no pneumatization of temporal bone showed a mean volume of $822+/-$ $50 \mathrm{~mm}^{3}$. This value indicates the volume of the middle ear cavity. Petrous apex cells and cells around labyrinthine were easily located when performing window thresholding and hence labyrinthine was used as a reference structure. In this study we found that the perilabyrinthine cells in group 3 were often extended to the posterior wall of the internal auditory canal. These air cells are of major concern in vestibular schwannoma surgery as they are known as the origin of CSF fistulae. [31] 
Apical cells are anatomically located anterior-medial to the internal auditory canal (IAC) and posterior medial to the carotid canal, again consistently present in group 3. These cells significantly contributed to the total volume of the temporal bone in the present study. When the carotid canal was used as the reference structure, group 3 cases (33\%) showed extensive pneumatization surrounding the carotid canal and the petrous apex region. During sub-temporal transpetrosal transtentorial approach surgery, the petrous apex region medial to the IAC is resected and it often becomes a route for CSF rhinorrhea. The pneumatized petrous apex is frequently responsible for CSF leakage after skull base surgery. Stieglitz et al. showed that a $10 \%$ increase in petrous bone pneumatization increased the risk of CSF fistulae by $26 \%$ [31].

The eustachian tube (ET) has important physiologic functions including pressure regulation and protection of the middle ear from infectious pathogens from the nasopharynx [19, 29]. ET dysfunction can be caused by a variety of mechanisms, including upper respiratory tract infections, sinusitis and adenoidal hypertrophy. The presence of peritubal cells and their communication with the ET were highly correlated with the degree of pneumatization in the present study. The relationship of peritubal cells to the bony segment of the eustachian tube plays a major role in development of CSF rhinorrhea after skull base surgery.

Temporal bones in our study showed direct communication of peritubal cells with the ET in $63.9 \%$ of the cases. The most common communication was at the posterior lateral part of the ET towards the middle ear cavity. ET is known as a common pathway for development of CSF rhinorrhea during translabyrinthine, suboccipital and middle cranial fossa surgery for acoustic neuromas. In a study conducted by Jen et al., it was stated that direct communication of peritubal cells with the osseous portion of ET plays an important role in the development of persistent CSF rhinorrhea after cerebellopontine angle surgery [19].

CBCT clearly showed the presence of air cells in the temporal bone due to high spatial resolution. Although there are many significant uses of $\mathrm{CBCT}$ in the imaging of the temporal bone, the low radiation dose which increases noise and gives poor density resolution of soft tissues, may restrict utilizing this imaging modality rather than MSCT and significantly affect its use for pre- and post-surgical evaluation of skull base surgeries. If the indication for using CBCT is correct it can be used to reduce radiation and as an inexpensive technique. Hodez et al. [16] showed that CBCT images of a well pneumatized middle ear and temporal bone are comparable 
to high resolution MSCT for visualizing ossicular erosions, traumatic lesions, osseous labyrinthine wall dehiscence and bony dysplasias. 


\section{Conclusion:}

This study showed that CBCT can be effectively used for imaging temporal bone air cavities. The mean volume of air cells in perilabyrinthine, middle ear, carotid and petrous regions was $1339 \mathrm{~mm}^{3}$. The petrous apex, labyrinthine and petrous segment of carotid canal are critical structures which can be located easily on CBCT scans and used as references to assess the pneumatization patterns. As an OMF radiologist it is important to define the normal appearances and patterns of the temporal bone pneumatization, and to identify potential pathologic changes. In a variety of temporal bone diseases and otitis media, pneumatization can be affected significantly; this study showed that analysis of air cells and their volume measurements can be done effectively utilizing CBCT.

As CBCT is being used widely, and may be approaching standard of care across the field of dentistry, especially for TMJ and orthodontic evaluation, it is important to identify early changes in the temporal bone, especially in the regions of inner ear and the central skull base, to make appropriate referrals to neuroradiologist and ENT specialists to avoid long term functional disability. 


\section{Future Directions:}

Chronic inflammation of the middle ear especially in children can suppress pneumatization of the temporal bone. It also causes bony erosion and disturbances in the ossicular chain. A comparative study of diseased bones vs. normal bones needs to be done to correctly identify changes in the pneumatization pattern and differences in the degree of pneumatization. 


\section{References}

1. Allam, A. F. (1969). Pneumatization of the temporal bone. Annals of Otology, Rhinology and Laryngology, 78(1), 49-64.

2. Apuhan, T., Yildirim, Y. S., \& Özaslan, H. (2011). Is there any developmental relationship between mastoid pneumatization and adenoid tissue volume? International Journal of Pediatric Otorhinolaryngology, 75(3), 415-419.

3. Austin, D. F. (1977). On the function of the mastoid. Otolaryngologic Clinics of North America, 10(3), 541-547.

4. Balzeau, A., \& Radovčić, J. (2008). Variation and modalities of growth and development of the temporal bone pneumatization in neandertals. Journal of Human Evolution, 54(5), 546-567.

5. Bast, T. H., \& Forester, H. B. (1939). Origin and distribution of air cells in the temporal bone. Arch Otolaryngol, 30, 183-205.

6. Çakli, H., Cingi, C., Ay, Y., Oghan, F., Ozer, T., \& Kaya, E. (2012). Use of cone beam computed tomography in otolaryngologic treatments. European Archives of Oto-RhinoLaryngology, 269(3), 711-720.

7. Carter, L. C., Haller, A. D., Calamel, A. D., \& Pfaffenbach, A. C. (1999). Zygomatic air cell defect (ZACD). prevalence and characteristics in a dental clinic outpatient population. Dentomaxillofacial Radiology, 28(2), 116-122.

8. Dahmani-Causse, M., Marx, M., Deguine, O., Fraysse, B., Lepage, B., \& Escudé, B. (2011). Morphologic examination of the temporal bone by cone beam computed tomography: Comparison with multislice helical computed tomography. European Annals of Otorhinolaryngology, Head and Neck Diseases, 128(5), 230-235.

9. Diamant, M. (1940). Otitis and pneumatization of the mastoid bone. Acta Otolaryngol, 41(SUPPL.), 1-149.

10. Gray's Anatomy $40^{\text {th }}$ edition ( $\left.\mathrm{p}-615\right)$

11. Habesoglu, T. E., Habesoglu, M., Toros, S. Z., Deveci, I., Surmeli, M., Sheidaei, S., et al. (2010). How does childhood otitis media change the radiological findings of the temporal bone? Acta Oto-Laryngologica, 130(11), 1225-1229.

12. Han, S. -., Song, M. H., Kim, J., Lee, W. -., \& Lee, H. -. (2007). Classification of temporal bone pneumatization based on sigmoid sinus using computed tomography. Clinical Radiology, 62(11), 1110-1118.

13. Hentona, H., Ohkubo, J., Tsutsumi, T., Tanaka, H., \& Komatsuzaki, A. (1994). Pneumatization of the petrous apex. Journal of Otolaryngology of Japan, 97(3), 450-456.

14. Hill, C. A. (2011). Ontogenetic change in temporal bone pneumatization in humans. Anatomical Record, 294(7), 1103-1115.

15. Hill, C. A., \& Richtsmeier, J. T. (2008). A quantitative method for the evaluation of three-dimensional structure of temporal bone pneumatization. Journal of Human Evolution, 55(4), 682-690. 
16. Hodez, C., Griffaton-Taillandier, C., \& Bensimon, I. (2011). Cone-beam imaging: Applications in ENT. European Annals of Otorhinolaryngology, Head and Neck Diseases, 128(2), 65-78.

17. IBM Corp. Released 2012. IBM SPSS Statistics for Windows, Version 21.0. Armonk, NY: IBM Corp.

18. Jen, A., Sanelli, P. C., Banthia, V., Victor, J. D., \& Selesnick, S. H. (2004). Relationship of petrous temporal bone pneumatization to the eustachian tube lumen. Laryngoscope, 114(4), 656-660.

19. Jen, A., Sanelli, P. C., Banthia, V., Victor, J. D., \& Selesnick, S. H. (2004). Relationship of petrous temporal bone pneumatization to the eustachian tube lumen. Laryngoscope, 114(4), 656-660.

20. Kenna, M. A. (1996). Embryology and developmental anatomy of the ear. Pediatric Otolaryngology, 1, 113-126.

21. Ladeira, D. B. S., Barbosa, G. L. R., Nascimento, M. C. C., Cruz, A. D., Freitas, D. Q., \& Almeida, S. M. (2013). Prevalence and characteristics of pneumatization of the temporal bone evaluated by cone beam computed tomography. International Journal of Oral and Maxillofacial Surgery, 42(6), 771-775.

22. Majdani, O., Thews, K., Bartling, S., Leinung, M., Dalchow, C., Labadie, R., et al. (2009). Temporal bone imaging: Comparison of flat panel volume CT and multisection CT. American Journal of Neuroradiology, 30(7), 1419-1424.

23. Palva, T., \& Palva, A. (1966). Size of the human mastoid air cell system. Acta OtoLaryngologica, 62(3), 237-251.

24. Palva, T., \& Ramsay, H. (2002). Fate of the mesenchyme in the process of pneumatization. Otology and Neurotology, 23(2), 192-199.

25. Redfors, Y. D., Gröndahl, H. G., Hellgren, J., Lindfors, N., Nilsson, I., \& Möller, C. (2012). Otosclerosis: Anatomy and pathology in the temporal bone assessed by multislice and cone-beam CT. Otology and Neurotology, 33(6), 922-927.

26. Richards, S. D., Saeed, S. R., Laitt, R., \& Ramsden, R. T. (2004). Hypercellularity of the mastoid as a cause of spontaneous pneumocephalus. Journal of Laryngology and Otology, 118(6), 474-476.

27. Sade, J. (1992). The correlation of middle ear aeration with mastoid pneumatization. the mastoid as a pressure buffer. European Archives of Oto-Rhino-Laryngology, 249(6), 301304.

28. Scarfe, W. C., Li, Z., Aboelmaaty, W., Scott, S. A., \& Farman, A. G. (2012). Maxillofacial cone beam computed tomography: Essence, elements and steps to interpretation. Australian Dental Journal, 57 Suppl 1, 46-60.

29. Seibert, J. W., \& Danner, C. J. (2006). Eustachian tube function and the middle ear. Otolaryngologic Clinics of North America, 39(6), 1221-1235.

30. Sherwood, R. J. (1995). The Hominid Temporal Bone: Ontogeny and Phylogenetic Implications, 
31. Stieglitz, L. H., Giordano, M., Gerganov, V., Raabe, A., Samii, A., Samii, M., et al. (2010). Petrous bone pneumatization is a risk factor for cerebrospinal fluid fistula following vestibular schwannoma surgery. Neurosurgery, 67(SUPPL. 2), ons509-ons515.

32. Tyndall, D. A., \& Matteson, S. R. (1987). The zygomatic air cell defect (ZACD) on panoramic radiographs. Oral Surgery Oral Medicine and Oral Pathology, 64(3), 373376.

33. Virapongse, C., Sarwar, M., \& Bhimani, S. (1985). Computed tomography of temporal bone pneumatization: 1. normal pattern and morphology. American Journal of Neuroradiology, 6(4), 551-559.

34. Xu, J., Reh, D. D., Carey, J. P., Mahesh, M., \& Siewerdsen, J. H. (2012). Technical assessment of a cone-beam CT scanner for otolaryngology imaging: Image quality, dose, and technique protocols. Medical Physics, 39(8), 4932-4942. 